\title{
On Some Interpolative Contractions of Suzuki Type Mappings
}

\author{
Andreea Fulga $\mathbb{D}^{1}$ and Seher Sultan Yeşilkaya $\mathbb{D}^{2}$ \\ ${ }^{1}$ Department of Mathematics and Computer Sciences, Universitatea Transilvania Brasov, Brasov, Romania \\ ${ }^{2}$ Division of Applied Mathematics, Thu Dau Mot University, Binh Duong Province, Vietnam
}

Correspondence should be addressed to Seher Sultan Yeşilkaya; yesilkaya@tdmu.edu.vn

Received 26 May 2021; Revised 15 June 2021; Accepted 30 June 2021; Published 23 July 2021

Academic Editor: Santosh Kumar

Copyright (c) 2021 Andreea Fulga and Seher Sultan Yeşilkaya. This is an open access article distributed under the Creative Commons Attribution License, which permits unrestricted use, distribution, and reproduction in any medium, provided the original work is properly cited.

The goal of this study is to propose a new interpolative contraction mapping by using an interpolative approach in the setting of complete metric spaces. We present some fixed point theorems for interpolative contraction operators using $w$-admissible maps which satisfy Suzuki type mappings. In addition, some results are given. These results generalize several new results present in the literature. Moreover, examples are provided to show the suitability of our given results.

\section{Introduction}

In 1922, Banach [1] proved his famous remarkable fixedpoint theorem; the result is known as the Banach contraction principle, which states that "Let $(\mathscr{K}, \mathrm{d})$ be a complete metric space and $\mathrm{S}: \mathscr{K} \longrightarrow \mathscr{K}$ be a contraction, then $S$ has a unique fixed point." The Banach contraction principle is one of the essential and most valuable theorems of analysis and is accepted as the main results of metric fixed-point theory. In the last century, the fixed point and its applications have been the subject of research by many authors in the literature, since it provides useful tools to solve many complex problems that have applications in different sciences like computer science, engineering, data science, physics, economics, game theory, and biosciences [2-7]. Due to several applications of "fixed point theory," researchers were motivated to further generalize it in different directions, by generalizing the contractive conditions underlying the space concept of completeness.

The background literature on the famous Banach contraction principle has been extended in various comprehensive directions by many researchers. One of the exciting generalizations was given by Kannan [8], which characterize the completeness of underlying metric spaces. Kannan introduced the following theorem.
Theorem 1. [8] Let $(\mathscr{K}, d)$ be a complete metric space. A mapping $S: \mathscr{K} \longrightarrow \mathscr{K}$ is said to be a Kannan contraction if there exists $\lambda \in[0,1 / 2)$ such that

$$
d(S v, S t) \leq \lambda(d(v, S v)+d(t, S t))
$$

for all $v, t \in \mathscr{K} \backslash$ Fix $(S)$. Then, $S$ posses a unique fixed point.

The Kannan theorem has been generalized in different aspects by many authors; one of the crucial generalizations was given by Karapinar in [9]. Karapinar [9] introduced the notion of an interpolative Kannan contraction mapping and proved the following:

A mapping $\mathrm{S}: \mathscr{K} \longrightarrow \mathscr{K}$ on $(\mathscr{K}, \mathrm{d})$ a complete metric space such that

$$
\mathrm{d}(\mathrm{S} v, \mathrm{~S} t) \leq \kappa[\mathrm{d}(v, \mathrm{~S} v)]^{\alpha} \cdot[\mathrm{d}(t, \mathrm{~S} t)]^{1-\alpha},
$$

where $\kappa \in[0,1)$ and $\alpha \in(0,1)$, for each $v, t \in \mathscr{K} \backslash \operatorname{Fix}(S)$. Then, $S$ has a unique fixed point in $\mathscr{K}$. Subsequently, Karapinar et al. [10] introduced the following notion of interpolative Ciric-Reich-Rus contractions.

Theorem 2 (see [10]). Let $(\mathscr{K}, p)$ be a partial metric space. The mapping $S: \mathscr{K} \longrightarrow \mathscr{K}$ is called an interpolative Ciric- 
Reich-Rus contraction if there exist $\lambda \in[0,1)$ and positive reals $\beta, \alpha>0$, with $\beta+\alpha<1$, such that

$$
p(S v, S t) \leq \lambda\left([p(v, t)]^{\beta} \cdot[p(v, S v)]^{\alpha} \cdot[p(t, S t)]^{1-\alpha-\beta}\right)
$$

for each $v, t \in \mathscr{K} \backslash$ Fix $(S)$. Then, the mapping $S$ has a fixed point in $\mathscr{K}$.

Afterward, this concept has been extended in different aspects, for example, [11-15].

Let $\Psi$ be the set of all nondecreasing self-mappings $\psi$ on $[0, \infty)$ such that $\sum_{\mathrm{r}=1}^{\infty} \psi^{\mathrm{r}}(z)<\infty$ for every $z>0$. Notice that for $\psi \in \Psi$, we have $\psi(0)=0$ and $\psi(z)<z$ for all $z>0$ (see $[16,17])$.

The concept of $w$-orbital admissible mappings was introduced by Popescu as a clarification of the concept of $\alpha$ -admissible mappings of Samet et al. [18].

Definition 3 (see [19]). Let $S$ be a self-map defined on $\mathscr{K}$ and $w: \mathscr{K} \times \mathscr{K} \longrightarrow[0, \infty)$ be a function. $S$ is said to be an $w$-orbital admissible if for all $v \in \mathscr{K}$, we have

$$
w(v, \mathrm{~S} v) \geq 1 \Rightarrow w\left(\mathrm{~S} v, \mathrm{~S}^{2} v\right) \geq 1
$$

In our appointed theorems, if the continuity of the involved contractive mappings is removed, to handle this defect, it is necessary that $(\mathscr{K}, \mathrm{d})$ be $w$-regular.

$(\mathfrak{R})$ A space $(\mathscr{K}, \mathrm{d})$ is defined as $w$-regular, if $\left\{v_{r}\right\}$ is a sequence in $\mathscr{K}$ such that $w\left(v_{r}, v_{r+1}\right) \geq 1$ for each $r$ and $v_{r} \longrightarrow \omega \in \mathscr{K}$ as $r \longrightarrow \infty$, then $w\left(v_{r}, \omega\right) \geq 1$ for all $r$.

Some curious results in this sense are found in the works in [20-24].

Another most interesting Banach contraction principle generalization was given by Suzuki $[25,26]$. He introduced a weaker notion of contraction and discussed the existence of some new fixed point theorems. Besides the famous theorem, Suzuki generalized also the results of Nemytzki [27] and Edelstein [28] for compact metric spaces. One of the recently popular topics in fixed point theory is addressing the existence of fixed points of Suzuki type mappings. As with many generalizations of the famous Banach theorems, Suzuki type generalization can be said to have many applications, such as in computer science [29], game theory [30], and biosciences [31] and in other areas of mathematical sciences such as in dynamic programming, integral equations, data dependence, and homotopy $[32,33]$. Subsequently, Popescu [34] has modified the nonexpansiveness situation with the weaker $C$-condition presented by Suzuki. Accordingly, the existence of fixed points of maps satisfying the $C$-condition has been extensively studied (see [35-38]). Karapinar et al. [39] introduced the definition of a nonexpansive mapping satisfying the $C$-condition:
Definition 4. A mapping $S$ on a metric space $(\mathscr{K}, d)$ satisfies the $C$-condition if

$$
\frac{1}{2} d(v, \mathrm{~S} v) \leq d(v, t) \Rightarrow d(\mathrm{~S} v, \mathrm{~S} t) \leq d(v, t)
$$

for each $v, t \in \mathscr{K}$.

\section{Main Results}

We start the section with the following essential definition:

Definition 5. Let $(\mathscr{K}, d)$ be a metric space. A mapping $S: \mathscr{K}$ $\longrightarrow \mathscr{K}$ is called an $w-\psi$-interpolative Kannan contraction of Suzuki type if there exist $\psi \in \Psi, w: \mathscr{K} \times \mathscr{K} \longrightarrow[0, \infty)$, and a real number $\beta \in[0,1)$, such that

$\frac{1}{2} \mathrm{~d}(v, \mathrm{~S} v) \leq \mathrm{d}(v, t) \Rightarrow w(v, t) \mathrm{d}(\mathrm{S} v, \mathrm{~S} t) \leq \psi\left([\mathrm{d}(v, \mathrm{~S} v)]^{\beta} \cdot[\mathrm{d}(t, \mathrm{~S} t)]^{1-\beta}\right)$,

for each $v, t \in \mathscr{K} \backslash \operatorname{Fix}(\mathrm{S})$.

Theorem 6. Let $(\mathscr{K}, d)$ be a complete metric space and $S: \mathscr{K} \longrightarrow \mathscr{K}$ be an $w-\psi$-interpolative Kannan contraction of the Suzuki type. Suppose that $S$ is an $w$-orbital admissible mapping and $w\left(v_{0}, S v_{0}\right) \geq 1$ for some $v_{0} \in \mathscr{K}$. Then, $S$ has a fixed point in $\mathscr{K}$ provided that at least one of the following conditions holds:

(a) $(\mathscr{K}, d)$ is w-regular

(b) $S$ is continuous

(c) $S^{2}$ is continuous and $w(v, S v) \geq 1$ where $v \in \operatorname{Fix}\left(S^{2}\right)$

Proof. Let $v_{0} \in \mathscr{K}$ such that $w\left(v_{0}, \mathrm{~S} v_{0}\right) \geq 1$ and $\left\{v_{r}\right\}$ be the sequence constructed by $S^{r}\left(v_{0}\right)=v_{r}$ for each positive integer $r$. Assuming that for some $r_{0} \in \mathbb{N}, v_{r_{0}}=v_{r_{0+1}}$, we get $v_{r_{0}}=\mathrm{S} v_{r_{0}}$, so $v_{r_{0}}$ is a fixed point of $S$. Then, $v_{r} \neq v_{r+1}$ for each positive integer $r$. As $S$ is $w$-orbital admissible, $w\left(v_{0}, \mathrm{~S} v_{0}\right)=w\left(v_{0}\right.$, $\left.v_{1}\right) \geq 1$ implies that $w\left(v_{1}, \mathrm{~S} v_{1}\right)=w\left(v_{1}, v_{2}\right) \geq 1$. Similarly, continuing this process, we have

$$
w\left(v_{r}, v_{r+1}\right) \geq 1
$$

Thereupon, choosing $v=v_{r-1}$ and $t=\mathrm{S} v_{r-1}$ in (6), we get

$$
\begin{gathered}
\frac{1}{2} d\left(v_{r-1}, S v_{r-1}\right)=\frac{1}{2} d\left(v_{r-1}, v_{r}\right) \leq d\left(v_{r-1}, v_{r}\right) \\
\Rightarrow d\left(v_{r}, v_{r+1}\right) \leq w\left(v_{r-1}, v_{r}\right) d\left(S v_{r-1}, S v_{r}\right) \\
\leq \psi\left(\left[d\left(v_{r-1}, S v_{r-1}\right)\right]^{\beta} \cdot\left[d\left(v_{r}, S v_{r}\right)\right]^{1-\beta}\right) \\
=\psi\left(\left[d\left(v_{r-1}, v_{r}\right)\right]^{\beta} \cdot\left[d\left(v_{r}, v_{r+1}\right)\right]^{1-\beta}\right) \\
<\left[d\left(v_{r-1}, v_{r}\right)\right]^{\beta} \cdot\left[d\left(v_{r}, v_{r+1}\right)\right]^{1-\beta},
\end{gathered}
$$


whence it follows that

$$
\left[d\left(v_{r}, v_{r+1}\right)\right]^{\beta}<\left[d\left(v_{r-1}, v_{r}\right)\right]^{\beta},
$$

or equivalent

$$
d\left(v_{r}, v_{r+1}\right)<d\left(v_{r-1}, v_{r}\right)
$$

Thus, on the one hand, it follows that the sequence $\left\{d\left(v_{r-1}, v_{r}\right)\right\}$ is a nonincreasing sequence with positive terms, so there exists $1 \geq 0$ such that $\lim _{r \longrightarrow \infty} d\left(v_{r-1}, v_{r}\right)=1$. On the other hand, combining (8) and (10) and keeping in mind that the function $\psi$ is nondecreasing, we obtain

$$
d\left(v_{r}, v_{r+1}\right) \leq \psi\left(d\left(v_{r-1}, v_{r}\right)\right) \leq \psi^{2}\left(d\left(v_{r-2}, v_{r-1}\right)\right) \leq \cdots \leq \psi^{r}\left(d\left(v_{0}, v_{1}\right)\right) .
$$

Now, applying the triangle inequality and using (11), for all $j \geq 1$, we get

$$
\begin{aligned}
d\left(v_{r}, v_{r+j}\right) \leq & d\left(v_{r}, v_{r+1}\right)+d\left(v_{r+1}, v_{r+2}\right)+\cdots+d\left(v_{r+j-1}, v_{r+j}\right) \\
\leq & \psi^{r}\left(d\left(v_{0}, v_{1}\right)+\psi^{r+1}\left(d\left(v_{0}, v_{1}\right)+\cdots+\psi^{r+j-1}\right.\right. \\
& \cdot\left(d\left(v_{0}, v_{1}\right)=\sum_{m=r}^{r+j-1} \psi^{m}\left(d\left(v_{0}, v_{1}\right)=P_{r+j-1}-P_{r-1},\right.\right.
\end{aligned}
$$

where $P_{k}=\sum_{m=0}^{k} \psi^{m}\left(d\left(v_{0}, v_{1}\right)\right)$. But, $\psi \in \Psi$, the series $\sum_{m=0}^{\infty}$ $\psi^{m}\left(d\left(v_{0}, v_{1}\right)\right)$ is convergent, so there exists a positive real number $P$ such that $\lim _{k \rightarrow \infty} P_{k}=P$. Consequently, letting $\mathrm{r}$, $j \longrightarrow \infty$ in the above inequality, we get

$$
d\left(v_{r}, v_{r+j}\right) \longrightarrow 0
$$

Therefore, $\left\{v_{r}\right\}$ is a Cauchy sequence, and taking into account the completeness of the space $(\mathscr{K}, \mathrm{d})$, it follows that there exists $\omega \in \mathscr{K}$ such that

$$
\lim _{r \longrightarrow \infty} v_{\mathrm{r}}=\omega,
$$

and we claim that this $\omega$ is a fixed point of $S$.

In case that the assumption (a) holds, we have $w\left(v_{r}, \omega\right)$ $\geq 1$, and we claim that

$$
\frac{1}{2} d\left(v_{r}, S v_{r}\right) \leq d\left(v_{r}, \omega\right)
$$

or

$$
\frac{1}{2} d\left(S v_{r}, S\left(S v_{r}\right)\right) \leq d\left(S v_{r}, \omega\right)
$$

for every $r \in \mathbb{N}$. Supposing

$$
\begin{gathered}
\frac{1}{2} d\left(v_{r}, S v_{r}\right)>d\left(v_{r}, \omega\right), \\
\frac{1}{2} d\left(S v_{r}, S\left(S v_{r}\right)\right)>d\left(S v_{r}, \omega\right),
\end{gathered}
$$

on the account of the triangle inequality, we have

$$
\begin{aligned}
d\left(v_{r}, v_{r+1}\right)= & d\left(v_{r}, S v_{r}\right) \leq d\left(v_{r}, \omega\right)+d\left(\omega, S v_{r}\right) \\
& <\frac{1}{2} d\left(v_{r}, S v_{r}\right)+\frac{1}{2} d\left(S v_{r}, S\left(S v_{r}\right)\right) \\
= & \frac{1}{2} d\left(v_{r}, v_{r+1}\right)+\frac{1}{2} d\left(v_{r+1}, v_{r+2}\right) \\
& \leq \frac{1}{2} d\left(v_{r}, v_{r+1}\right)+\frac{1}{2} d\left(v_{r}, v_{r+1}\right)=d\left(v_{r}, v_{r+1}\right),
\end{aligned}
$$

which is a contradiction. Thereupon, for every $r \in \mathbb{N}$, either

$$
\frac{1}{2} d\left(v_{r}, S v_{r}\right) \leq d\left(v_{r}, \omega\right)
$$

or

$$
\frac{1}{2} d\left(S v_{r}, S\left(S v_{r}\right)\right) \leq d\left(S v_{r}, \omega\right),
$$

holds. In the case that (19) holds, we obtain

$$
\begin{gathered}
d\left(v_{r+1}, S \omega\right) \leq w\left(v_{r}, \omega\right) d\left(S v_{r}, S \omega\right) \leq \psi\left[d \left(\left[d\left(v_{r}, S v_{r}\right)\right]^{\beta} \cdot[d(\omega, S \omega)]^{1-\beta}\right.\right. \\
=\psi\left[\left[d\left(v_{r}, v_{r+1}\right)\right]^{\beta} \cdot[d(\omega, S \omega)]^{1-\beta}<\left[d\left(v_{r}, v_{r+1}\right)\right]^{\beta} \cdot[d(\omega, S \omega)]^{1-\beta} .\right.
\end{gathered}
$$

If the second condition, (20), holds, we have

$$
\begin{aligned}
& d\left(v_{r+2}, S \omega\right) \leq w\left(v_{r+1}, \omega\right) d\left(S^{2} v_{r}, S \omega\right) \\
& \quad \leq \psi\left[d \left(\left[d\left(S v_{r}, S^{2} v_{r}\right)\right]^{\beta} \cdot[d(\omega, S \omega)]^{1-\beta}\right.\right. \\
& =\psi\left[\left[d\left(v_{r+1}, v_{r+2}\right)\right]^{\beta} \cdot[d(\omega, S \omega)]^{1-\beta}\right. \\
& \quad<\left[d\left(v_{r+1}, v_{r+2}\right)\right]^{\beta} \cdot[d(\omega, S \omega)]^{1-\beta} .
\end{aligned}
$$

Therefore, letting $r \longrightarrow \infty$ in (21) and (22), we get that $d(\omega, S \omega)=0$, that is, $\omega=\mathrm{S} \omega$.

In the case that the assumption (b) is true, that is, the mapping $S$ is continuous,

$$
S \omega=\lim _{r \longrightarrow \infty} S v_{r}=\lim _{r \longrightarrow \infty} v_{r+1}=\omega .
$$

If the last assumption, (c), holds, as above, we have $S^{2} \omega$ $=\lim _{r \longrightarrow \infty} S^{2} v_{r}=\lim _{r \longrightarrow \infty} v_{r+2}=\omega$ and we want to show that also $S \omega=\omega$. Supposing on the contrary, that $\omega \neq S \omega$, since

$$
\frac{1}{2} d\left(S \omega, S^{2} \omega\right)=\frac{1}{2} d(S \omega, \omega) \leq d(S \omega, \omega),
$$


by (6), we get

$$
\begin{aligned}
d(\omega, S \omega) & \leq w(S \omega, \omega) d\left(S^{2} \omega, S \omega\right) \leq \psi\left(\left[d\left(S \omega, S^{2} \omega\right)\right]^{\beta} \cdot\left[d(\omega, S \omega)^{1-\beta}\right]\right) \\
& <[d(S \omega, \omega)]^{\beta} \cdot\left[d(\omega, S \omega)^{1-\beta}\right]=d(S \omega, \omega),
\end{aligned}
$$

which is a contradiction. Consequently, $\omega=S \omega$, that is, $\omega$ is a fixed point of the mapping $S$.

Example 7. Let $\mathscr{K}=[0,3]$ and $d: \mathscr{K} \times \mathscr{K} \longrightarrow[0,+\infty)$ be the usual distance on $\mathbb{R}$. Consider the mapping $S: \mathscr{K} \longrightarrow \mathscr{K}$ be defined as

$$
S v= \begin{cases}\frac{4}{5}, & \text { if } v \in[0,1] \\ \frac{1}{3}, & \text { if } v \in(1,2] \\ \frac{\sqrt[3]{v^{3}+6 v+\ln (4-v)}}{5}, & \text { if } v \in(2,3] .\end{cases}
$$

Let also $w: \mathscr{K} \times \mathscr{K} \longrightarrow[0, \infty)$, where

$$
w(v, t)= \begin{cases}2, & \text { if } v, t \in[0,1] \\ v^{2}+t^{2}, & \text { if } v, t \in(1,2) \\ 1, & \text { if } v=0, t=3 \\ 0, & \text { otherwise. }\end{cases}
$$

We remark that the space is not regular since, for example, considering the sequence $\left\{v_{\mathrm{r}}\right\}$, with $v_{r}=(r+8) /(2 r+4)$ we have $v_{r} \longrightarrow 1 / 2$ as $r \longrightarrow \infty, w\left(v_{r}, v_{r+1}\right)=v_{r}^{2}+v_{r+1}^{2} \leq 1$, but $w$ $\left(v_{\mathrm{r}}, 1 / 2\right)=0$. On the other hand, the mapping $S$ is not continuous, but since $S^{2}=4 / 5$, we have that $S^{2}$ is a continuous mapping. Let the function $\psi \in \Psi$ defined as $\psi(z)=z / 3$ and we choose $\beta=1 / 9$. Thus, we have to check that (6) holds. We have to consider the following cases:

(1) For $v, t \in[0,1]$, respectively, $v, t \in(1,2)$, we have $d$ $(\mathrm{S} v, \mathrm{~S} t)=0$, so $(6)$ holds

(2) For $v=0$ and $t=3$

$$
\begin{aligned}
\frac{1}{2} d(0, \text { S } 0) & =\frac{2}{5}<3=d(0,3) \Rightarrow w(0,3) d(S 0, \text { S3 }) \\
& =0,088621339 \leq 0,678785019 \\
& =\frac{1}{3}(0.8)^{1 / 9} \cdot(2,288621339)^{8 / 9} \\
& =\psi\left([d(0, \text { S0 })]^{1 / 9} \cdot[\mathrm{d}(3, S 3)]^{8 / 9}\right)
\end{aligned}
$$

(3) All other cases are not interesting because $w(v, t)=0$

Consequently, the assumptions of Theorem 6 being satisfied, it follows that the mapping $S$ has a fixed point, which is $v=4 / 5$.
Corollary 8. Let $(\mathscr{K}, d)$ be a complete metric space and $S$ be a self-mapping on $\mathscr{K}$, such that,

$$
\frac{1}{2} d(v, S v) \leq d(v, t) \text { implies } d(S v, S t) \leq \psi\left([d(v, S v)]^{\beta} \cdot[d(t, S t)]^{1-\beta}\right)
$$

for each $v, t \in \mathscr{K} \backslash$ Fix $(S)$, where $\psi \in \Psi$ and $\beta \in[0,1)$. Then, $S$ possesses a fixed point in $\mathscr{K}$.

Proof. Theorem 6 is sufficient to get $w(v, t)=1$ for the proof.

Moreover, taking $\psi(z)=z \kappa$, with $\kappa \in[0,1)$ in Corollary (8), we obtain the following consequence.

Corollary 9. Let $(\mathscr{K}, d)$ be a complete metric space and $S$ be a self-mapping on $\mathscr{K}$, such that

$$
\frac{1}{2} d(v, S v) \leq d(v, t) \text { implies } d(S v, S t) \leq \kappa[d(v, S v)]^{\beta} \cdot[d(t, S t)]^{1-\beta},
$$

for each $v, t \in \mathscr{K} \backslash \operatorname{Fix}(S)$, where $\beta \in[0,1)$. Then, the mapping $S$ possesses a fixed point in $\mathscr{K}$.

Definition 10. Let $(\mathscr{K}, d)$ be a metric space. The mapping $S: \mathscr{K} \longrightarrow \mathscr{K}$ is called an $w-\psi$-interpolative Cirić-Reich-Rus contraction of Suzuki type if there exist $\psi \in \Psi, w: \mathscr{K} \times \mathscr{K}$ $\longrightarrow[0, \infty)$, and positive reals $\beta, \alpha>0$, with $\beta+\alpha<1$, such that

$$
\begin{aligned}
& \frac{1}{2} \mathrm{~d}(v, \mathrm{~S} v) \leq \mathrm{d}(v, t) \Rightarrow w(v, t) \mathrm{d}(\mathrm{S} v, \mathrm{~S} t) \\
& \quad \leq \psi\left([\mathrm{d}(v, t)]^{\beta} \cdot[\mathrm{d}(v, \mathrm{~S} v)]^{\alpha} \cdot[\mathrm{d}(t, \mathrm{~S} t)]\right)^{1-\alpha-\beta}
\end{aligned}
$$

for each $v, t \in \mathscr{K} \backslash \operatorname{Fix}(S)$.

Theorem 11. Let $(\mathscr{K}, d)$ be a complete metric space and the mapping $S: \mathscr{K} \longrightarrow \mathscr{K}$ be an $w-\psi$-interpolative CirićReich-Rus contraction of the Suzuki type. Suppose that $S$ is $w$-orbital admissible and $w\left(v_{0}, S v_{0}\right) \geq 1$ for some $v_{0} \in \mathscr{K}$. If $(\mathscr{K}, d)$ is $w$-regular or either

(1) $S$ is continuous or

(2) $S^{2}$ is continuous and $w(S \omega, \omega) \leq 1$ for any $v \in \operatorname{Fix}\left(S^{2}\right)$, then the mapping $S$ has a fixed point in $\mathscr{K}$

Proof. Let $v_{0} \in \mathscr{K}$ satisfy $w\left(v_{0}, \mathrm{~S} v_{0}\right) \geq 1$ and $\left\{v_{r}\right\}$ be the sequence defined by $S^{r}\left(v_{0}\right)=v_{r}$ for each positive integer $r$. If $v_{r_{0}}=v_{r_{0+1}}$ for some $r_{0} \in \mathbb{N}$, we get $v_{r_{0}}=S v_{r_{0}}$, that means $v_{r_{0}}$ is a fixed point of $S$. Then, we can assume that $v_{r} \neq v_{r+1}$ for each positive integer $r$. Moreover, due to the assumption that $S$ is $w$-orbital admissible, as in the previous proof, we 
have

$$
w\left(v_{r}, v_{r+1}\right) \geq 1
$$

By letting $v=v_{r-1}$ and $t=\mathrm{S} v_{r-1}=v_{r}$ in (31), we obtain

$$
\begin{aligned}
& \frac{1}{2} d\left(v_{r-1}, S v_{r-1}\right)=\frac{1}{2} d\left(v_{r-1}, v_{r}\right) \\
& \quad \leq d\left(v_{r-1}, v_{r}\right) \Rightarrow d\left(v_{r}, v_{r+1}\right) \\
& \quad \leq w\left(v_{r-1}, v_{r}\right) d\left(S v_{r-1}, S v_{r}\right) \\
& \quad \leq \psi\left(\left[d\left(v_{r-1}, v_{r}\right)\right]^{\beta} \cdot\left[d\left(v_{r-1}, S v_{r-1}\right)\right]^{\alpha} \cdot\left[d\left(v_{r}, S v_{r}\right)\right]^{1-\alpha-\beta}\right) \\
& =\psi\left(\left[d\left(v_{r-1}, v_{r}\right)\right]^{\beta} \cdot\left[d\left(v_{r-1}, v_{r}\right)\right]^{\alpha} \cdot\left[d\left(v_{r}, v_{r+1}\right)\right]^{1-\alpha-\beta}\right),
\end{aligned}
$$

then, using $\psi(z)<z$ for every $z>0$.

$$
d\left(v_{r}, v_{r+1}\right) \leq\left[d\left(v_{r-1}, v_{r}\right)\right]^{\beta+\alpha} \cdot\left[d\left(v_{r}, v_{r+1}\right)\right]^{1-\alpha-\beta},
$$

or equivalent

$$
\left[d\left(v_{r}, v_{r+1}\right)\right]^{\alpha+\beta}<\left[d\left(v_{r-1}, v_{r}\right)\right]^{\alpha+\beta} .
$$

So,

$$
d\left(v_{r}, v_{r+1}\right)<d\left(v_{r-1}, v_{r}\right)
$$

for every $r \in \mathbb{N}$. Therefore, the positive sequence $\left\{d\left(v_{r-1}, v_{r}\right)\right\}$ is decreasing. Eventually, by (33), we have

$$
d\left(v_{r}, v_{r+1}\right) \leq \psi\left(d\left(v_{r-1}, v_{r}\right)\right)
$$

and by repeating this process, we find that

$$
d\left(v_{r}, v_{r+1}\right) \leq \psi^{r}\left(d\left(v_{0}, v_{1}\right)\right) .
$$

We assert that $\left\{v_{r}\right\}$ is a fundamental sequence in $(\mathscr{K}, \mathrm{d})$. Thus, using the triangle inequality with (38), we can write

$$
\begin{aligned}
& d\left(v_{r}, v_{r+l}\right) \leq d\left(v_{r}, v_{r+1}\right)+d\left(v_{r+1}, v_{r+2}\right)+\cdots+d\left(v_{r+l-1}, v_{r+l}\right) \\
& \quad \leq \psi^{r} d\left(v_{0}, v_{1}\right)+\psi^{r+1}\left(d\left(v_{0}, v_{1}\right)\right)+\cdots+\psi^{r+l-1}\left(d\left(v_{0}, v_{1}\right)\right) \\
& \quad \leq \sum_{k=r}^{\infty} \psi^{k}\left(d\left(v_{0}, v_{1}\right)\right) .
\end{aligned}
$$

Taking $r \longrightarrow \infty$ in (39), we deduce that $\left\{v_{r}\right\}$ is a fundamental sequence in $(\mathscr{K}, \mathrm{d})$, and using the completeness $(\mathscr{K}, \mathrm{d})$, there exists $\omega \in \mathscr{K}$ such that

$$
\lim _{r \longrightarrow \infty} \mathrm{d}\left(v_{r}, \omega\right)=0
$$

We claim that the point $\omega$ is a fixed point of $S$. In the case of the space $(\mathscr{K}, \mathrm{d})$ being $w$-regular and $\left\{v_{r}\right\}$ verifies (32), that is, $w\left(v_{r}, v_{r+1}\right) \geq 1$ for every $r \in \mathbb{N}$, we get $w\left(v_{r}\right.$, $\omega) \geq 1$. On the other hand, we know (see the proof of
Theorem 6) that either

$$
\frac{1}{2} d\left(v_{r}, S v_{r}\right) \leq d\left(v_{r}, \omega\right)
$$

or

$$
\frac{1}{2} d\left(S v_{r}, S\left(S v_{r}\right)\right) \leq d\left(S v_{r}, \omega\right)
$$

holds, for every $r \in \mathbb{N}$. If (41) is holds, we obtain

$$
\begin{gathered}
d\left(v_{r+1}, S \omega\right) \leq w\left(v_{r}, \omega\right) d\left(S v_{r}, S \omega\right) \\
\leq \psi\left[d\left(v_{r}, \omega\right)\right]^{\beta} \cdot\left[d\left(v_{r}, S v_{r}\right)\right]^{\alpha} \cdot[d(\omega, S \omega)]^{1-\alpha-\beta}, \\
=\psi\left[d\left(v_{r}, \omega\right)\right]^{\beta} \cdot\left[d\left(v_{r}, v_{r+1}\right)\right]^{\alpha} \cdot[d(\omega, S \omega)]^{1-\alpha-\beta} \\
\left.\quad<d\left(v_{r}, \omega\right)\right]^{\beta} \cdot\left[d\left(v_{r}, v_{r+1}\right)\right]^{\alpha} \cdot[d(\omega, S \omega)]^{1-\alpha-\beta} .
\end{gathered}
$$

Letting $r \longrightarrow \infty$ in the above inequality, we get that $d(\omega, \mathrm{S} \omega)=0$, that is, $\omega=S \omega$. If the second condition (42) is true, we get that $\omega$ is a fixed point $S$ by a similar argument.

Furthermore, if the $w$-regular of $(\mathscr{K}, \mathrm{d})$ is removed and, instead, $S$ is continuous, we get that $S$ has a fixed point in $\mathscr{K}$, because

$$
\omega=\lim _{r \longrightarrow \infty} v_{r+1}=\lim _{r \longrightarrow \infty} S v_{r}=S\left(\lim _{r \longrightarrow \infty} v_{r}\right)=S \omega .
$$

Finally, if the mapping $S$ is such that $S^{2}$ is continuous, we easily obtain $S^{2} \omega=\omega$. Supposing that $S \omega \neq \omega$, since $w(S \omega, \omega) \leq 1$ for any $v \in \operatorname{Fix}\left(S^{2}\right)$ and $(1 / 2) d\left(S \omega, S^{2}\right.$ $\omega)=(1 / 2) d(S \omega, \omega) \leq d(S \omega, \omega)$, we have

$$
\begin{aligned}
& d(\omega, S \omega)= d\left(S^{2} \omega, S \omega\right) \leq w(S \omega, \omega) d\left(S^{2} \omega, S \omega\right) \\
& \leq \psi\left([d(S \omega, \omega)]^{\alpha} \cdot\left[d\left(S \omega, S^{2} \omega\right)\right]^{\beta} \cdot[d(\omega, S \omega)]^{1-\alpha-\beta}\right) \\
&<[d(S \omega, \omega)]^{\alpha} \cdot[d(S \omega, \omega)]^{\beta} \cdot[d(\omega, S \omega)]^{1-\alpha-\beta}=[d(\omega, S \omega)]
\end{aligned}
$$

That is a contradiction. Thereupon, $S \omega=\omega$.

Example 12. Let $\mathscr{K}=\{0,1 / 8,1 / 4,1 / 2,1\}, d: \mathscr{K} \times \mathscr{K} \longrightarrow[0$, $+\infty), d(v, t)=|v-t|$, and $S: \mathscr{K} \longrightarrow \mathscr{K}$, where $S 0=S(1 / 2)$ $=1 / 8, S(1 / 8)=1 / 2$, and $S 1=S(1 / 4)=1 / 4$. Consider the function $w: \mathscr{K} \times \mathscr{K} \longrightarrow[0, \infty)$,

$$
w(v, t)= \begin{cases}1, & \text { if }(v, t) \in\left\{(0,1),\left(0, \frac{1}{2}\right)\right\}, \\ t+1, & \text { if }(v, t) \in \mathscr{K} \cup\left\{\frac{1}{4}\right\}, \\ 0, & \text { otherwise, }\end{cases}
$$

let $\psi \in \Psi, \psi(z)=2 z / 5$, and the real constants $\alpha=\beta=1 / 3$. 
Taking into account the definition of the function $w$, the only interesting situations are for $v=0, y=1 / 2$, respectively, $v=$ $0, y=1$. For the first case, we have

$$
\begin{aligned}
\frac{1}{2} d(0, S 0)= & \frac{1}{2} d\left(0, \frac{1}{8}\right)=\frac{1}{16}<\frac{1}{2}=d\left(0, \frac{1}{2}\right) \\
& \Rightarrow w\left(0, \frac{1}{2}\right) d\left(S 0, S \frac{1}{2}\right)=0 \\
& \left.\leq \psi\left(\left[d\left(0, \frac{1}{2}\right)\right]^{1 / 3} \cdot[d(0, S 0)]^{1 / 3}\right) \cdot\left[d\left(\frac{1}{2}, S \frac{1}{2}\right)\right]^{1 / 3}\right) .
\end{aligned}
$$

For the second case,

$$
\begin{aligned}
\frac{1}{2} d(0, S 0) & =\frac{1}{2} d\left(0, \frac{1}{8}\right)=\frac{1}{16}<1=d(0,1) \Rightarrow w(0,1) d(S 0, S 1) \\
& =\frac{1}{8} \leq 0,158740105=\frac{2}{5} \cdot\left(\frac{1}{8}\right)^{1 / 3} \cdot\left(\frac{3}{4}\right)^{1 / 3} \\
& =\psi\left([d(0,1)]^{1 / 3} \cdot[d(0, S 0)]^{1 / 3} \cdot[d(1, S 1)]^{1 / 3}\right)
\end{aligned}
$$

Definition 13. Let $(\mathscr{K}, d)$ be a metric space. The mapping $S: \mathscr{K} \longrightarrow \mathscr{K}$ is called an $\psi$-interpolative Cirić-Reich-Rus contraction of Suzuki type if there exist $\psi \in \Psi$ and the constants $\beta, \alpha>0$, with $\beta+\alpha<1$, such that

$$
\begin{aligned}
& \frac{1}{2} d(v, S v) \leq d(v, t) \Rightarrow d(S v, S t) \\
& \quad \leq \psi\left([d(v, t)]^{\beta} \cdot[d(v, S v)]^{\alpha} \cdot[d(t, S t)]^{1-\alpha-\beta}\right),
\end{aligned}
$$

for each $v, t \in \mathscr{K} \backslash \operatorname{Fix}(S)$.

Theorem 14. Let $(\mathscr{K}, d)$ be a complete metric space and the mapping $S: \mathscr{K} \longrightarrow \mathscr{K}$ be an $\psi$-interpolative Ćirić-ReichRus contraction of the Suzuki type. Then, the mapping $S$ has a fixed point in $\mathscr{K}$.

Proof. Put $w(v, t)=1$ in Theorem 11 .

Definition 15. Let $(\mathscr{K}, d)$ be a metric space. A mapping $S$ $: \mathscr{K} \longrightarrow \mathscr{K}$ is called an interpolative Cirić-Reich-Rus contraction of the Suzuki type if there exist $\kappa \in[0,1)$ and positive reals $\beta, \alpha>0$, with $\beta+\alpha<1$, such that

$$
\begin{aligned}
& \frac{1}{2} \mathrm{~d}(v, \mathrm{~S} v) \leq \mathrm{d}(v, t) \Rightarrow \mathrm{d}(\mathrm{S} v, \mathrm{~S} t) \leq \kappa[\mathrm{d}(v, t)]^{\beta} \cdot[\mathrm{d}(v, \mathrm{~S} v)]^{\alpha} \\
& \cdot[\mathrm{d}(t, \mathrm{~S} t)]^{1-\alpha-\beta}
\end{aligned}
$$

for each $v, t \in \mathscr{K} \backslash \operatorname{Fix}(\mathrm{S})$.

Theorem 16. Let $(\mathscr{K}, d)$ be a complete metric space and $S$ $: \mathscr{K} \longrightarrow \mathscr{K}$ be an interpolative Cirić-Reich-Rus contraction of the Suzuki type. Therefore, S has a fixed point in $\mathscr{K}$.
Proof. Put $\psi(z)=\kappa z$, for all $z>0$, in Theorem 14 .

\section{Conclusions}

In this manuscript, we introduce new concepts on completeness of $w$ - $\psi$-interpolative Kannan contraction of Suzuki type and $w$ - $\psi$-interpolative Ćirić-Reich-Rus contraction of Suzuki type mappings in metric space. We prove the existence of some fixed point theorems for mappings these concepts. Further, we obtain some fixed point results and give examples to show that the new results are applicable. Interpolation contraction, which is generalized from the Kannan type contraction, is a new and interesting contraction in fixed point theory, and different interpolation contractions of Suzuki type studies can be obtained by combining it with a Suzuki type contraction in the future. Additionally, these proposed contractions can be generalized in other well-known spaces and can give new fixed point results.

\section{Data Availability}

No data were used to support this study.

\section{Conflicts of Interest}

The authors declare that there is no conflict of interest regarding the publication of this paper.

\section{Authors' Contributions}

Both authors contributed equally to this work. Both authors have read and approved the final manuscript.

\section{References}

[1] S. Banach, "Sur les opérations dans les ensembles abstraits et leur application aux équations intégrales," Fundamenta Mathematicae, vol. 3, pp. 133-181, 1922.

[2] H. H. Bauschke, R. S. Burachik, P. L. Combettes, V. Elser, D. R. Luke, and H. Wolkowicz, Fixed Point Algorithms for Inverse Problems in Science and Engineering, Optimization and its Applications, Springer, New York, NY, USA, 1st edition, 2011.

[3] H. el-Dessouky and S. Bingulac, "A fixed point iterative algorithm for solving equations modeling the multi- stage flash desalination process," Computer Methods in Applied Mechanics and Engineering, vol. 141, no. 1-2, pp. 95-115, 1997.

[4] R. Levy, "Fixed point theory and structural optimization," Engineering Optimization, vol. 17, no. 4, pp. 251-261, 1991.

[5] N. Saleem, J. Vujaković, W. U. Baloch, and S. Radenović, "Coincidence point results for multivalued Suzuki type mappings using $\theta$-contraction in b-metric spaces," Mathematics, vol. 7, no. 11, p. 1017, 2019.

[6] E. Karapinar, A. Fulga, and R. P. Agarwal, "A survey: F-contractions with related fixed point results," Journal of Fixed Point Theory and Applications, vol. 22, no. 3, pp. 1-58, 2020.

[7] A. Panwar and Reena, "Approximation of fixed points of a multivalued $\rho$-quasi-nonexpansive mapping for newly defined hybrid iterative schemeApproximation of fixed points of a multivalued $\rho$-quasi-nonexpansive mapping for newly defined hybrid iterative scheme," Journal of Interdisciplinary Mathematics, vol. 22, no. 4, pp. 593-607, 2019. 
[8] R. Kannan, "Some results on fixed point," Bulletin of the Calcutta Mathematical Society, vol. 60, pp. 71-76, 1968.

[9] E. Karapınar, "Revisiting the Kannan type contractions via interpolation," Advances in the Theory of Nonlinear Analysis and its Application, vol. 2, no. 2, pp. 85-87, 2018.

[10] E. Karapinar, R. Agarwal, and H. Aydi, "Interpolative ReichRus-Ćirić type contractions on partial metric spaces," Mathematics, vol. 6, no. 11, p. 256, 2018.

[11] Y. U. Gaba and E. Karapinar, "A new approach to the interpolative contractions," Axioms, vol. 8, no. 4, p. 110, 2019.

[12] H. Aydi, E. Karapinar, and A. F. R. L. de Hierro, “ $\omega$-interpolative Cirić-Reich-Rus-type contractions,” Mathematics, vol. 7, no. 1, p. 57, 2019.

[13] E. Karapınar, O. Alqahtani, and H. Aydi, "On interpolative Hardy-Rogers type contractions," Symmetry, vol. 11, no. 1, p. 8, 2019.

[14] E. Karapinar and R. P. Agarwal, "Interpolative Rus-ReichĆirić type contractions via simulation functions," Analele Universitatii "Ovidius" Constanta - Seria Matematica, vol. 27, no. 3, pp. 137-152, 2019.

[15] P. Gautam, L. M. S. Ruiz, and S. Verma, "Fixed point of interpolative Rus-Reich-Ćirić contraction mapping on rectangular quasi-partial b-metric space," Symmetry, vol. 13, no. 1, p. 32, 2021.

[16] I. A. Rus, Generalized Contractions and Applications, Cluj University Press, Clui-Napoca, Romania, 2001.

[17] R. P. Agarwal, E. Karapınar, D. O’Regan, and A. F. RoldánLópez-de-Hierro, Fixed Point Theory in Metric Type Spaces, Springer, Cham, Switzerland, 2015.

[18] B. Samet, C. Vetro, and P. Vetro, "Fixed point theorems for $\alpha$ $\psi$-contractive type mappings," Nonlinear Analysis: Theory, Methods \& Applications, vol. 75, no. 4, pp. 2154-2165, 2012.

[19] O. Popescu, "Some new fixed point theorems for $\alpha$-Geraghty contraction type maps in metric spaces," Fixed Point Theory and Applications, vol. 2014, no. 1, 2014.

[20] H. Afshari, S. Kalantari, and H. Aydi, "Fixed point results for generalized $\alpha-\psi$-Suzuki-contractions in quasi-b-metric-like spaces," Asian-European Journal of Mathematics, vol. 11, no. 1, p. 1850012, 2018.

[21] H. Aydi, E. Karapınar, and B. Samet, "Fixed points for generalized $(\alpha, \psi)$-contractions on generalized metric spaces," Journal of Inequalities and Applications, vol. 2014, no. 1, 2014.

[22] C. M. Chen, A. Abkar, S. Ghods, and E. Karapınar, "Fixed point theory for the a-admissible Meir-Keeler-type set contractions having KKM property on almost convex sets," Applied Mathematics \& Information Sciences, vol. 11, no. 1, pp. 171-176, 2017.

[23] E. Karapınar and B. Samet, "Generalized $\alpha, \psi$-contractive type mappings and related fixed point theorems with applications," Abstract and Applied Analysis, vol. 2012, pp. 1-793417, 2012.

[24] E. Karapinar, "Discussion on $(\alpha, \psi)$-contractions on generalized metric spaces," Abstract and Applied Analysis, vol. 2014, Article ID 962784, 2014.

[25] T. Suzuki, "Some results on recent generalization of Banach contraction principle," in Proceedings of the 8th International Conference on Fixed Point Theory and Its Applications, pp. 751-761, Chiang Mai, Thailand, 2007.

[26] T. Suzuki, "A generalized Banach contraction principle that characterizes metric completeness," Proceedings of the American Mathematical Society, vol. 136, no. 5, pp. 1861-1869, 2008.
[27] V. V. Nemytzki, "Fixed point method in analysis," Uspekhi Matematicheskikh Nauk, vol. 1, pp. 141-174, 1936.

[28] M. Edelstein, "On fixed and periodic points under contractive mappings," Journal of the London Mathematical Society, vol. s1-37, no. 1, pp. 74-79, 1962.

[29] P. Salimi and E. Karapınar, "Suzuki-Edelstein type contractions via auxiliary functions," Mathematical Problems in Engineering, vol. 2013, Article ID 648528, 8 pages, 2013.

[30] H. Aydi, M. A. Barakat, Z. D. Mitrović, and V. Šešum-Čavić, "A Suzuki-type multivalued contraction on weak partial metric spaces and applications," Journal of inequalities and applications, vol. 2018, no. 1, 2018.

[31] F. A. Rihan, D. Baleanu, S. Lakshmanan, and R. Rakkiyappan, "On fractional SIRC model with Salmonella bacterial infection," Abstract and Applied Analysis, vol. 2014, Article ID 136263, 9 pages, 2014.

[32] N. Saleem, M. Abbas, B. Ali, and Z. Raza, "Fixed points of Suzuki-type generalized multivalued (f, $\theta, \mathrm{L})$-almost contractions with applications," Filomat, vol. 33, no. 2, pp. 499-518, 2019.

[33] M. Abbas, B. Ali, and C. Vetro, "A Suzuki type fixed point theorem for a generalized multivalued mapping on partial Hausdorff metric spaces," Topology and its Applications, vol. 160, no. 3, pp. 553-563, 2013.

[34] O. Popescu, "Two generalizations of some fixed point theorems," Computers \& Mathematcs with Applications, vol. 62, no. 10, pp. 3912-3919, 2011.

[35] T. Suzuki, "A new type of fixed point theorem in metric spaces," Nonlinear Analysis: Theory, Methods \& Applications, vol. 71, no. 11, pp. 5313-5317, 2009.

[36] E. Karapınar and K. Taş, "Generalized (C)-conditions and related fixed point theorems," Computers \& Mathematics with Applications, vol. 61, no. 11, pp. 3370-3380, 2011.

[37] E. Karapınar, "Remarks on Suzuki (C)-condition," in Dynamical Systems and Methods, A. Luo, J. Machado, and D. Baleanu, Eds., pp. 227-243, Springer, New York, 2012.

[38] R. S. Adiguzel, U. Aksoy, E. Karapinar, and I. M. Erhan, “On the solutions of fractional differential equations via Geraghty type hybrid contractions," Mathematical Methods in the Applied Sciences, vol. 20, no. 2, 2021.

[39] E. Karapınar, I. M. Erhan, and U. Aksoy, "Weak $\psi$-contractions on partially ordered metric spaces and applications to boundary value problems," Boundary Value Problems, vol. 2014, no. 1, 2014. 